\title{
Effect of relative humidity on the peeling behavior of a thin film on a rigid substrate
}

\author{
Zhilong Peng, ${ }^{1}$ Cong Wang, ${ }^{2}$ Yazheng Yang, ${ }^{1}$ and Shaohua Chen ${ }^{1, *}$ \\ ${ }^{1}$ Institute of Advanced Structure Technology, Beijing Institute of Technology, Beijing 100081, China \\ ${ }^{2}$ LNM, Institute of Mechanics, Chinese Academy of Sciences, Beijing 100190, China \\ (Received 28 April 2016; revised manuscript received 10 July 2016; published 7 September 2016)
}

\begin{abstract}
Inspired by gecko adhesion in humid environments, a modified Kendall's model is established in order to investigate the effect of relative humidity on the interfacial peeling behavior of a thin film adhering on a rigid substrate. When the humidity is less than $90 \%$, a monolayer of water molecules adsorbed on the substrate surface induces a strong disjoining pressure at the interface. As a result, the steady-state peel-off force between the thin film and substrate is significantly enhanced. When the humidity is greater than $90 \%$, water molecules condense into water droplets. Four different peeling models are established on this occasion, depending on the surface wettability of the film and substrate. It is found that the steady-state peel-off force is influenced by the water meniscus in a complicated manner, which is either enhanced or reduced by the water capillarity comparing to that predicted by the classical Kendall's model, i.e., a dry peeling model. It should be noted that, at the vicinity of the wetting transition, the peel-off force of the four models can be reduced to an identical one, which means the four peeling models can transit from one to another continuously. The present model, as an extension of the classical Kendall's one, should be useful not only for understanding gecko adhesion in humid environments, but also for analyzing interface behaviors of a film-substrate system in real applications.
\end{abstract}

DOI: 10.1103/PhysRevE.94.032801

\section{INTRODUCTION}

The interface behavior of film-substrate systems, as a very interesting topic, has been attracting considerable attention due to their ubiquitous application in modern industries and practical engineering. One of the most widely used techniques of characterizing interfacial properties is the peel test. Due to its simplicity and easy operation, the peel test has become one of the most efficient methods to assess the mechanical properties of interfaces and has been widely applied in many research fields, such as coating technology, microelectromechanical systems, adhesive tapes, and biomimetic adhesion [1-3].

In the past decades, numerous experimental and theoretical works have been carried out to investigate the peeling behavior of thin films on substrates [4-8]. For an elastic thin film adhesively contacting a rigid substrate, the classical Kendall's peeling model is widely adopted [5], which provides a direct method to derive the interface properties, including the adhesion toughness and adhesion strength, through measuring the peel-off force. Kendall's model was later extended to analyze the peeling behavior of elastic-plastic thin films [6], viscoelastic films [9-11], and heterogeneous films [12,13]. Based on the minimum potential principle, the whole peeling process from initial debonding to a steady-state stage of an elastic thin film on a smooth and rough substrate has been studied, considering the effect of the bending stiffness of thin films [14-16]. Some other representative theories of thin films peeling from substrates include the beam model and the cohesive zone model, in which the peeled film was considered as a slender beam $[6,7]$ or the cracked interface was regarded as a cohesive zone [17,18].

Recently, as a typical application, the peeling model is widely adopted in the field of biological and bioinspired adhesion [3,19-24]. Many climbing animals or

\footnotetext{
*chenshaohua72@hotmail.com
}

insects evolve amazing reversible adhesion mechanisms. For example, geckos' climbing ability depends on the fascinating hierarchical adhesive structure, which consists of millions of setae. Each seta further branches into hundreds of spatulae about $200 \mathrm{~nm}$ in width and length [25,26]. Each spatula looks like a nano thin film, which adheres on a substrate with van der Waals force. Accumulation of a large number of molecular forces could support a gecko's weight. The classical Kendall's peeling model has been adopted to study the reversible adhesion mechanism of geckos [20,24,27]. The peeling model with a finite-length film was further developed by Tian et al. [19] and Peng et al. [21] since the real profile of gecko spatulae looks more like a nanofilm with a finite-length scale.

All the above investigations focus on the peeling behavior of a thin film on a dry substrate. The effect of relative humidity on the peeling behavior of a thin film is still an open question. One of our previous works considered the effect of environmental humidity on the interface adhesion of film-substrate systems, but only the normal adhesion force was studied [22]. How the environmental humidity affects the peeling behavior of a thin film on a substrate is still unclear.

In this paper, the influence of environmental humidity on the peeling behavior of an elastic film adhering on a rigid substrate is investigated, in which the peel-off force of the film detached from the substrate is mainly considered. The main aim of this paper is to investigate the effect of environmental humidity on the interface adhesion, which is an extension of the classical Kendall's model [5]. Different models are established according to the varied relative humidity inducing a different stable form of water membrane. When the relative humidity is less than $90 \%$, a monolayer of water molecules (water membrane) will be adsorbed on the substrate. The corresponding model is analyzed in Sec. II. Water molecules can also condense into a liquid meniscus near the interface crack tip forming four different wetting models depending on the wettability of the film and substrate if the relative 
humidity is larger than $90 \%$. The corresponding theoretical model is given and analyzed in Sec. III. The effect of relative humidity on the peel-off force in each model is discussed in Sec. IV and conclusions are made in Sec. V. The theoretical finding should be useful for understanding geckos' adhesion in their natural living habitat and helpful for the design of film-substrate systems working in humid environments.

\section{EFFECT OF WATER MEMBRANE}

Huber et al. [28] found a monolayer of water molecules adsorbed on substrate surfaces when the environmental humidity is less than $88 \%$. DelRio et al. [29] also found a monolayer water membrane or a bimolecular layer adsorbed on a substrate when the relative humidity is about $70 \%$. Therefore, a peeling model of an elastic film adhering on a rigid substrate is established as shown in Fig. 1(a) for the case of relative humidity smaller than $90 \%$ or so, where a monolayer water membrane is assumed to be adsorbed on the interface. The area

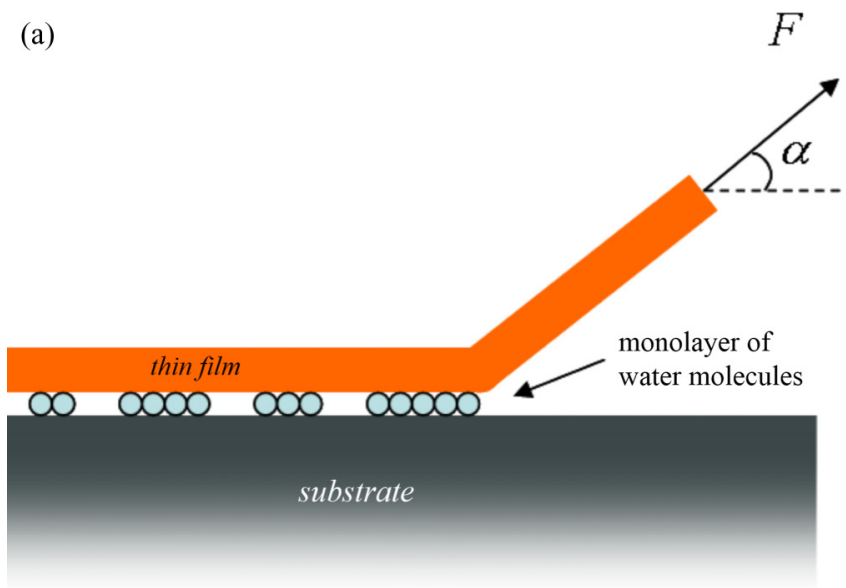

(b)

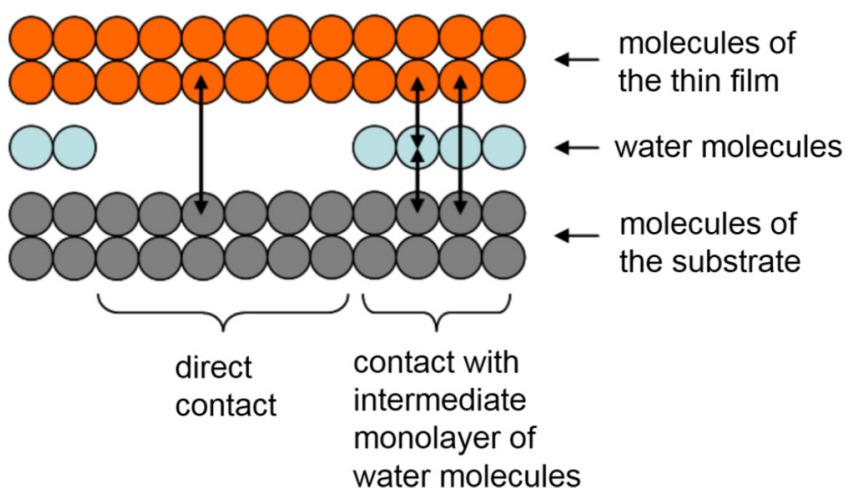

FIG. 1. Schematics of a thin film in adhesive contact with a rigid substrate with an intermediate monolayer of water molecules. (a) The thin film is peeled by an external force $F$ with a peeling angle $\alpha$ and the water coverage ratio is $\rho$; (b) on the interface, the interaction includes not only the force between atoms of solid materials, but also the one between water molecules and atoms of solid materials, i.e., the disjoining pressure. coverage of water membrane is $\rho$. The elastic film is peeled by an external force $F$ with a peeling angle $\alpha$.

It has been indicated that the disjoining pressure between the liquid molecules and solid surfaces is so strong that the liquid membrane lies flat on the surfaces looking like a very thin liquid layer [30]. There are three independent components of the disjoining pressure, in which van der Waals interaction between the liquid layer and solid surface dominates [30]. When a thin film contacts a substrate with an intermediate monolayer of water as shown in Fig. 1(a), the total adhesion force of the interface includes not only the interaction force between the thin film and substrate but also the attraction force between water molecules and the solid surfaces [Fig. 1(b)].

At a thermal equilibrium state, the water coverage ratio $\rho$ on the rigid substrate is a function of the relative humidity, which can be obtained from the Langmuir's adsorption isotherm $[22,28]$ :

$$
\rho=\frac{\mathrm{RH}}{\mathrm{RH}+\exp \left(-E_{a} / \kappa_{B} T\right)} \approx \mathrm{RH} \exp \left(\frac{E_{a}}{\kappa_{B} T}\right) \approx 1.22 \mathrm{RH},
$$

where RH denotes the relative humidity. $E_{a}$ is the adsorption energy, which is typically much less than the thermal energy $\kappa_{B} T$ at room temperature. The interface adhesion energy $w(D)$ between two adjacent surfaces with a separation distance $D$ can be derived by volume integration of the commonly used Lennard-Jones potential $V(r)=4 \varepsilon\left[(\sigma / r)^{12}-(\sigma / r)^{6}\right]$. Here, $\varepsilon$ is a parameter determining the depth of the potential well. $\sigma$ is a length scale parameter that determines the position of the minimum potential. $r$ is an atom-to-atom distance [23]. Then, we have

$w(D)=\int_{V} V(r) d V=\frac{A_{H}}{12 \pi D_{0}^{2}}\left[\left(\frac{D_{0}}{D}\right)^{2}-\frac{1}{4}\left(\frac{D_{0}}{D}\right)^{8}\right]$,

where $A_{H}=4 \pi^{2} \varepsilon \rho_{A} \rho_{B} \sigma^{6}$ is the Hamaker constant and $D_{0}=(2 / 15)^{1 / 6} \sigma$ the equilibrium distance between two contact surfaces.

The effective adhesion energy between the thin film and substrate with an intermediate water membrane at the interface can be expressed as

$$
\begin{aligned}
\Delta \gamma_{\mathrm{eff}}= & (1-\rho) w_{S S-\mathrm{dry}}(D)+\rho\left[w_{S S-\text { wet }}(D)+w_{S L}(d)\right] \\
= & \frac{(1-\rho) A_{H-\mathrm{dry}}+\rho A_{H-\mathrm{wet}}}{12 \pi D_{0}^{2}}\left[\left(\frac{D_{0}}{D}\right)^{2}-\frac{1}{4}\left(\frac{D_{0}}{D}\right)^{8}\right] \\
& +\frac{\rho A_{H-S L}}{12 \pi d_{0}^{2}}\left[\left(\frac{d_{0}}{d}\right)^{2}-\frac{1}{4}\left(\frac{d_{0}}{d}\right)^{8}\right]
\end{aligned}
$$

where $w_{S S-\text { dry }}$ represents the adhesion energy between two solid surfaces with an air interval. $w_{S S-\text { wet }}$ is the adhesion energy between two solid surfaces with an intermediate water membrane. $w_{S L}$ denotes the adhesion energy between the water film and solid surfaces. $D$ and $d$ are the solid-solid and solid-liquid surface distances, respectively. $D_{0}$ and $d_{0}$ are the corresponding equilibrium distances. $A_{H-S L}, A_{H-\mathrm{dry}}$, and $A_{H-\text { wet }}$ denote Hamaker constants for the solid-liquid 
interaction, solid-solid interaction with an air interval, and the one with an intermediate water membrane, respectively.

According to the classical Kendall's model [5], it is convenient for us to obtain the peel-off force $F$ as a function of the peeling angle $\alpha$. The only difference is to replace the adhesion energy in Kendall's model with the effective one $\Delta \gamma_{\text {eff }}$ in Eq. (3),

$$
\frac{F}{E h}=-(1-\cos \alpha)+\sqrt{(1-\cos \alpha)^{2}+2 \frac{\Delta \gamma_{\mathrm{eff}}}{E h}},
$$

where $E$ is the Young's modulus of the elastic film. $h$ denotes the film's thickness.

\section{EFFECT OF WATER DROPLET}

Mate and his co-worker have found experimentally that the disjoining pressure between the liquid molecules and the solid surface will decrease when the thickness of the liquid film increases [30,31]. For a liquid monolayer spreading on a substrate, the liquid molecules would gain more energy from the attractive interaction than they would gain in entropy by adopting a bulk-like conformation. However, when the relative humidity is more than $90 \%$, the liquid film is thick enough. As a result, the disjoining pressure becomes so weak that entropy would cause the liquid molecules to condense into a bulk-like conformation [22,30,31]. Therefore, a water droplet forms. When the surface tension of film $\gamma_{1}$ is less than the sum of the surface tension of water $\gamma$ and the interface tension of water and film $\gamma_{1 L}$, i.e., $\gamma_{1}<\gamma+\gamma_{1 L}$, the water droplet stays on the film with a contact angle $\theta_{1}$. Similarly, the water droplet lies on the substrate with a contact angle $\theta_{2}$, i.e., $\gamma_{2}<$ $\gamma+\gamma_{2 L}$, where $\gamma_{2}$ is the surface tension of the substrate; $\gamma_{2 L}$ denotes the interface tension of water and substrate. A water meniscus condenses near the interface crack tip between the thin film and substrate as shown in Fig. 2(a), which abides by the Young-Laplace equation,

$$
\begin{aligned}
& \gamma \cos \theta_{1}+\gamma_{1 L}=\gamma_{1} \quad 0<\theta_{1}<\pi ; \\
& \gamma \cos \theta_{2}+\gamma_{2 L}=\gamma_{2} \quad 0<\theta_{2}<\pi .
\end{aligned}
$$

If $\gamma_{1} \geqslant \gamma+\gamma_{1 L}$ and $\gamma_{2} \geqslant \gamma+\gamma_{2 L}$, it is favorable for the liquid spreading on the solid surface with $\theta_{1}=\theta_{2}=0$ as shown in Fig. 2(b) [32,33]. In our model, the water droplet spreads on the substrate surface and the whole peeled-off film. As for the case of $\gamma_{1}<\gamma+\gamma_{1 L}$ and $\gamma_{2} \geqslant \gamma+\gamma_{2 L}$ or the one with $\gamma_{1} \geqslant \gamma+\gamma_{1 L}$ and $\gamma_{2}<\gamma+\gamma_{2 L}$, the water droplet would completely spread on one surface but partially wet the other one as shown in Figs. 2(c) and 2(d), respectively.

\section{A. Case A: The case of $\gamma_{1}<\gamma+\gamma_{1 L}$ and $\gamma_{2}<\gamma+\gamma_{2 L}$}

As a stable water droplet condenses near the interface crack tip in Fig. 2(a), the force exerted on the film by the water meniscus consists of two parts: the surface tension of water $\gamma$ and the Laplace pressure force $\Delta P$ as shown in Fig. 3(a). At a thermal equilibrium state, the mean curvature of the meniscus $\kappa$ can be determined from the Kelvin equation [34],

$$
\kappa=\frac{R T}{\gamma V} \ln \left(\frac{p}{p_{0}}\right),
$$
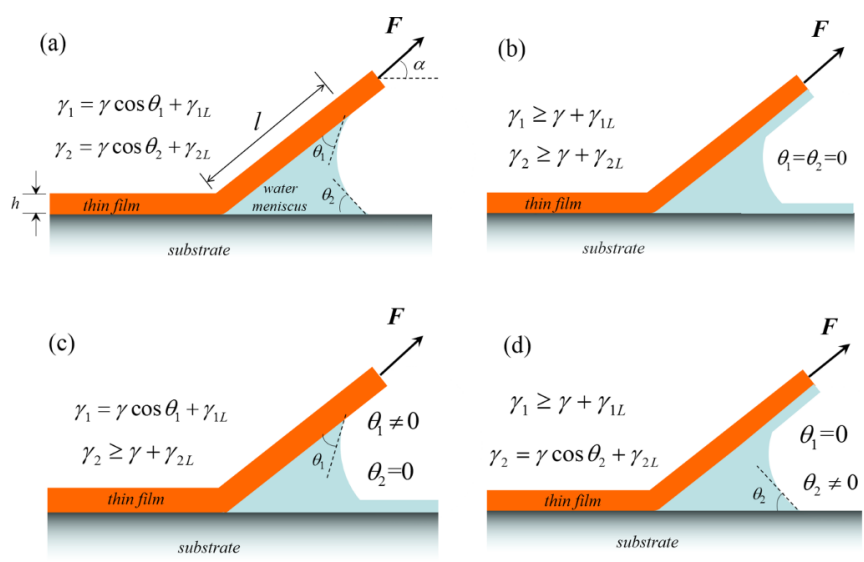

FIG. 2. Schematics of a thin film peeled from a rigid substrate when the relative humidity is larger than $90 \%$. The water molecules will condense into water droplets. (a) The water droplet forms a water meniscus near the interface crack tip with two contact angles $\theta_{1}$ and $\theta_{2}$ on the elastic film and rigid substrate, respectively. The wetted length of the film is $l$; (b) the water droplet spreads on surfaces of the film and substrate with vanishing contact angles $\theta_{1}$ and $\theta_{2}$; (c) the water droplet wets the film surface with a given contact angle $\theta_{1}$ but completely spreads on the substrate surface with a vanishing contact angle $\theta_{2} ;$ (d) the water droplet wets the substrate surface with a given contact angle $\theta_{2}$ but completely spreads on the film surface with a vanishing contact angle $\theta_{1}$.

where $R$ is the universal gas constant; $T$ is the absolute temperature; $V$ is the molar volume; $p_{0}$ is the saturated vapor pressure of water meniscus at $T ; p$ is the ambient pressure outside the curved surface. $p / p_{0}$ denotes the relative humidity.

The surface tension of water meniscus acting on the film leads to the strain in different parts of the film,

$$
\begin{aligned}
& \varepsilon_{0}=0 \text { in the adhesive interface region, } \\
& \varepsilon_{1}=\frac{F-\gamma \cos \theta_{1}}{E h} \text { in the free and wetted region, } \\
& \varepsilon_{2}=\frac{F}{E h} \text { in the free and dry region. }
\end{aligned}
$$

Assuming a virtual infinitesimal peeled-off length $\Delta s$ during the steady-state peeling process as shown in Fig. 3(b), the liquid bridge will move in a steady-state manner with the propagation of the interface crack tip. The shape of the meniscus as well as the film's wetting length remains unchanged in this stage.

The work done by the externally peeling force $F$ can be written as

$$
\begin{aligned}
W_{F} & =F\left(1+\varepsilon_{1}-\cos \alpha\right) \Delta s \\
& =F(1-\cos \alpha) \Delta s+\frac{F\left(F-\gamma \cos \theta_{1}\right)}{E h} \Delta s .
\end{aligned}
$$

Precisely, one part of the length $\Delta s$ should deform under both the external force $F$ and the capillary force $\gamma$ as shown in Eq. (8), and the other part is only subjected to the force $F$ as shown in Eq. (9), though the peeling length $\Delta s$ itself is infinitesimal. Here, for simplicity, we assume the whole infinitesimal peeling length $\Delta s$ deforms under the coupled effect of the external force $F$ and the capillary one $\gamma$. 

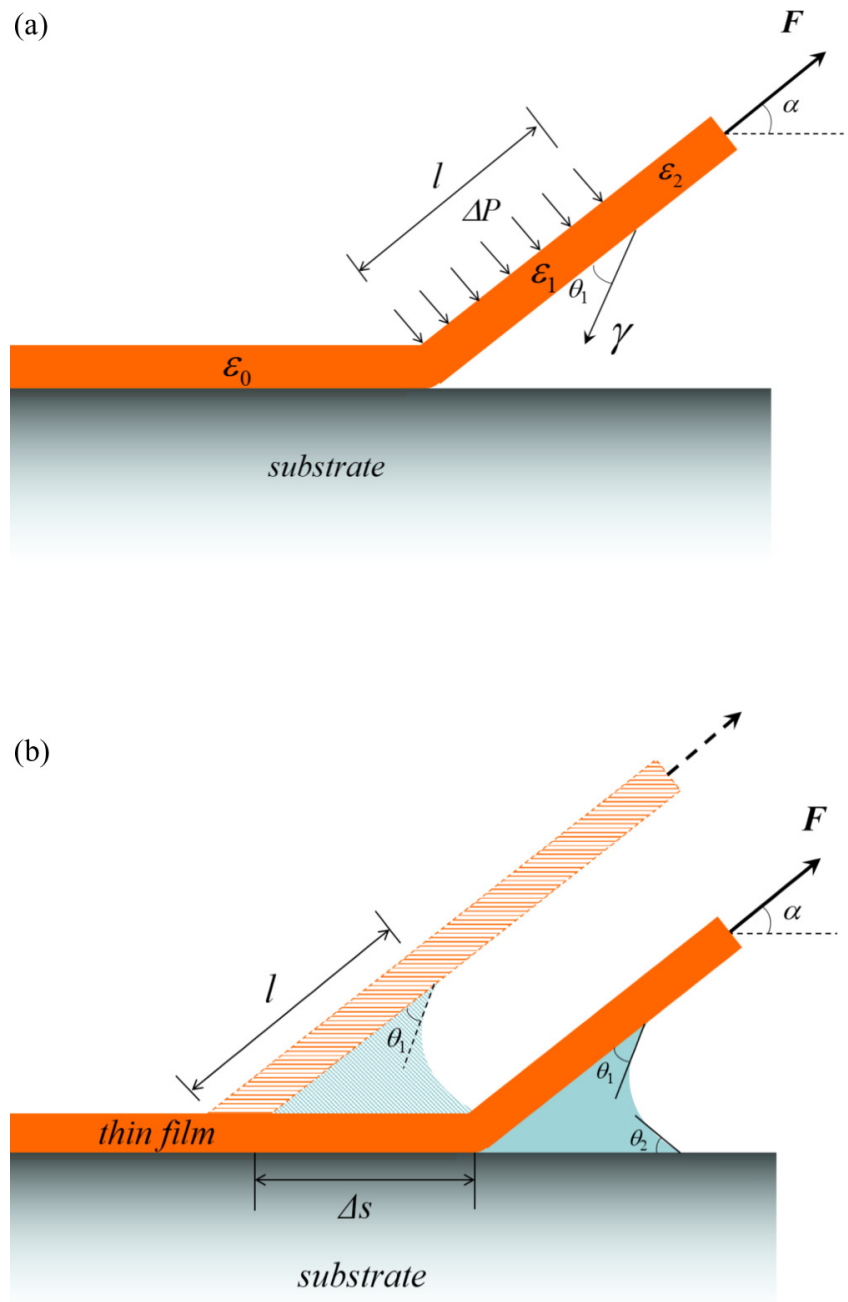

FIG. 3. The peeling model with a water meniscus near the interface crack tip. (a) In the region of the wetted film part, the water meniscus induces a surface tension of water $\gamma$ and Laplace pressure $\Delta P$ acting on the wetted film part; (b) during a steady-state peeling stage, the water meniscus moves along with the interface crack in a steady-state manner without any change. $\Delta s$ denotes an infinitesimal peeling length.

The work contributed by the force induced by water meniscus $W_{\text {capillary }}$ includes two parts: $W_{\gamma}$ done by the surface tension of water and $W_{\Delta P}$ contributed by the Laplace pressure,

$$
\begin{aligned}
W_{\gamma} & =-\gamma \cos \theta_{1}\left(1+\varepsilon_{1}-\cos \alpha\right) \Delta s-\gamma \sin \theta_{1} \Delta s \sin \alpha \\
& =-\gamma\left[\cos \theta_{1}\left(1+\varepsilon_{1}\right)-\cos \left(\theta_{1}+\alpha\right)\right] \Delta s \\
W_{\Delta P} & =\int_{0}^{l-\left(1+\varepsilon_{1}\right) \Delta s}\left(\Delta p \vec{n} \cdot \vec{x}_{F 1}\right) d l \\
& \approx-\Delta p l \sin \alpha \Delta s .
\end{aligned}
$$

Considering the Young-Laplace equation $\Delta p=\gamma / r$ and the geometrical relationship $l \sin \alpha=r \cos \left(\theta_{1}+\alpha\right)+$ $r \cos \theta_{2}$, where $r$ is the mean radius of curvature of the liquid meniscus and $l$ is the wetting length on the film as shown in Fig. 3(b), we have

$$
W_{\Delta P}=-\gamma\left[\cos \left(\theta_{1}+\alpha\right)+\cos \theta_{2}\right] \Delta s .
$$

Therefore,

$$
W_{\text {capillary }}=W_{\Delta P}+W_{\gamma}=-\gamma\left[\cos \theta_{1}\left(1+\varepsilon_{1}\right)+\cos \theta_{2}\right] \Delta s .
$$

Substituting the Young's equations $\gamma \cos \theta_{1}=\gamma_{1}-\gamma_{1 L}$ and $\gamma \cos \theta_{2}=\gamma_{2}-\gamma_{2 L}$ into Eq. (14), we can rewrite Eq. (14) as

$$
\begin{aligned}
W_{\text {capillary }}= & -\left(\gamma_{1}-\gamma_{1 L}\right) \Delta s-\left(\gamma_{2}-\gamma_{2 L}\right) \Delta s \\
& -\left(\gamma_{1}-\gamma_{1 L}\right) \frac{F-\left(\gamma_{1}-\gamma_{1 L}\right)}{E h} \Delta s .
\end{aligned}
$$

From the above equation, one can see that the work done by the water meniscus equals the variation of surface energy in the trailing region of the liquid meniscus.

Summation of the variation of the surface energy in the leading region of the liquid meniscus and the elastic energy stored in the film is

$$
\begin{aligned}
\Delta E_{\text {surface }}^{\text {leading }}+\Delta E_{\text {elastic }}= & \left(\gamma_{1 L}+\gamma_{2 L}-\gamma_{12}\right) \Delta s \\
& +\frac{\left(F-\gamma \cos \theta_{1}\right)^{2}}{2 E h} \Delta s .
\end{aligned}
$$

Thus the following energy conservation equation,

$$
W_{F}+W_{\text {capillary }}=\Delta E_{\text {surface }}^{\text {leading }}+\Delta E_{\text {elastic }},
$$

yields the governing equation of the peel-off force,

$$
F(1-\cos \alpha)+\frac{\left[F-\left(\gamma_{1}-\gamma_{1 L}\right)\right]^{2}}{2 E h}-\Delta \gamma=0,
$$

where $\Delta \gamma=\gamma_{1}+\gamma_{2}-\gamma_{12}$ is the interface adhesion energy between the film and substrate. $\gamma_{12}$ is the interface energy. The closed-form solution of the peel-off force can be derived as

$$
\frac{F}{E h}=\cos \alpha-1+\sqrt{(1-\cos \alpha)^{2}-2 \frac{\left(\gamma_{1}-\gamma_{1 L}\right)(1-\cos \alpha)-\Delta \gamma}{E h}}+\frac{\gamma_{1}-\gamma_{1 L}}{E h} .
$$

Using the relationship $\Delta \gamma_{1 L}=\gamma_{1}+\gamma-\gamma_{1 L}$, we can rewrite Eq. (19) as

$$
\frac{F}{E h}=\cos \alpha-1+\sqrt{(1-\cos \alpha)^{2}-2 \frac{\left(\Delta \gamma_{1 L}-\gamma\right)(1-\cos \alpha)-\Delta \gamma}{E h}}+\frac{\Delta \gamma_{1 L}-\gamma}{E h},
$$

where $\Delta \gamma_{1 L}$ corresponds to the adhesion energy between the water meniscus and thin film. Here, $\Delta \gamma_{1 L}$ should satisfy the condition of $\Delta \gamma_{1 L}<2 \gamma$ due to the wetting configuration in Case A. 


\section{B. Case B: The case of $\gamma_{1} \geqslant \gamma+\gamma_{1 L}$ and $\gamma_{2} \geqslant \gamma+\gamma_{2 L}$}

When surface tensions of both the film and substrate are not less than the sum of the water surface tension and the solidliquid interface tension, the water droplet is favorable to spread on the solid surfaces with $\theta_{1}=\theta_{2}=0$ [32,33] from the energy point of view as shown in Fig. 2(b). During the steady-state peeling process, the peeling force added on the film is balanced by both the tensile stress in the film and the surface tension of water. In this case, Eqs. (7) and (8) are still valid with contact angles $\theta_{1}=\theta_{2}=0$. Similar to Sec. III A, assuming a virtual infinitesimal peeling length $\Delta s$ leads to variations of the surface energy $\Delta E_{\text {surface, }}$, elastic energy stored in the film $\Delta E_{\text {elastic }}$, and work done by the externally peeling force $W_{F}$,

$$
\begin{aligned}
\Delta E_{\text {surface }} & =\left[\left(\gamma_{1 L}+\gamma\right)+\left(\gamma_{2 L}+\gamma\right)-\gamma_{12}\right] \Delta s+\gamma \varepsilon_{1} \Delta s, \\
\Delta E_{\text {elastic }} & =\frac{(F-\gamma)^{2}}{2 E h} \Delta s, \\
W_{F} & =F(1-\cos \alpha) \Delta s+\frac{F(F-\gamma)}{E h} \Delta s .
\end{aligned}
$$

Energy conservation among the above three energies yields the governing equation

$$
F(1-\cos \alpha)+\frac{(F-\gamma)^{2}}{2 E h}=\gamma_{1 L}+\gamma_{2 L}+2 \gamma-\gamma_{12}
$$

which results in the peel-off force,

$$
\begin{aligned}
\frac{F}{E h} & =\cos \alpha-1+\sqrt{(1-\cos \alpha)^{2}+2 \frac{\gamma_{1 L}+\gamma_{2 L}-\gamma_{12}+\gamma(1+\cos \alpha)}{E h}}+\frac{\gamma}{E h} \\
& =\cos \alpha-1+\sqrt{(1-\cos \alpha)^{2}+2 \frac{\Delta \gamma-\Delta \gamma_{1 L}-\Delta \gamma_{2 L}+\gamma(3+\cos \alpha)}{E h}}+\frac{\gamma}{E h} .
\end{aligned}
$$

Here $\Delta \gamma_{1 L}$ and $\Delta \gamma_{2 L}$ should satisfy conditions of $\Delta \gamma_{1 L} \geqslant 2 \gamma$ and $\Delta \gamma_{2 L} \geqslant 2 \gamma$.

One should note that the interfacial adhesion energy $\Delta \gamma$ depends on the interface mode mixity [35]; the details can be found in Hutchinson and Suo [36]. However, it was found that, when the effect of mixed-mode fracture on the peeling behavior of a thin film adhering on a dry substrate is considered, all the results are qualitatively similar to that with a constant interfacial adhesion energy $[8,15,27]$. Without loss of generality, the interfacial adhesion energy is assumed to be a constant in the present paper for simplicity.

\section{Case C: The case of $\gamma_{1}<\gamma+\gamma_{1 L}$ and $\gamma_{2} \geqslant \gamma+\gamma_{2 L}$}

When the surface tension of the film is less than the sum of the water surface tension and the film-liquid interface tension, but with the surface tension of the substrate less than the sum of the water surface tension and the substrate-liquid interface tension, the water droplet will be pinned on the film surface with a given contact angle $\theta_{1}$ but completely spreads on the substrate surface with a vanishing contact angle $\theta_{2}$ as shown in Fig. 2(c). During the steady-state peeling process with a virtual infinitesimal peeling length $\Delta s$, the work done by the externally peeling force $W_{F}$ and the elastic energy stored in the film $\Delta E_{\text {elastic }}$ have the same expressions as given in Eqs. (10) and (16). The variation of the total surface energy $\Delta E_{\text {surface }}$ includes the variation of the surface energy in the trailing edge and that in the leading edge, which can be expressed as

$$
\begin{aligned}
\Delta E_{\text {surface }}= & \left(\gamma_{1}+\gamma_{2 L}+\gamma-\gamma_{12}\right) \Delta s+\gamma \cos \theta_{1} \varepsilon_{1} \Delta s \\
= & \left(\Delta \gamma+2 \gamma-\Delta \gamma_{2 L}\right) \Delta s \\
& +\left(\gamma_{1}-\gamma_{1 L}\right) \frac{F-\left(\gamma_{1}-\gamma_{1 L}\right)}{E h} \Delta s .
\end{aligned}
$$

Energy conservation among the work done by the external peeling force, the elastic energy stored in the film, and the variation of the total surface energy leads to the governing equation of the peel-off force,

$$
\frac{\left[F-\left(\gamma_{1}-\gamma_{1 L}\right)\right]^{2}}{2 E h}+F(1-\cos \alpha)-\Delta \gamma-2 \gamma+\Delta \gamma_{2 L}=0 .
$$

The peel-off force can be obtained as

$$
\frac{F}{E h}=\cos \alpha-1+\sqrt{(1-\cos \alpha)^{2}-2 \frac{\left(\Delta \gamma_{1 L}-\gamma\right)(1-\cos \alpha)-\left(\Delta \gamma+2 \gamma-\Delta \gamma_{2 L}\right)}{E h}}+\frac{\Delta \gamma_{1 L}-\gamma}{E h} .
$$




\section{Case D: The case of $\gamma_{1} \geqslant \gamma+\gamma_{1 L}$ and $\gamma_{2}<\gamma+\gamma_{2 L}$}

Contrary to the above Case $\mathrm{C}$, when the surface tension of the film is not less than the sum of the water surface tension and the film-liquid interface tension, but with the surface tension of the substrate less than the sum of the water surface tension and the substrate-liquid interface tension, the water droplet will completely spread on the film surface with a vanishing contact angle $\theta_{1}$ but is pinned on the substrate surface with a contact angle $\theta_{2}$ as shown in Fig. 2(d). During the steady-state peeling process, we assume the film is peeled off a virtual infinitesimal length $\Delta s$. The elastic energy stored in the film $\Delta E_{\text {elastic }}$ and the work done by the externally peeling force $W_{F}$ should have the same expressions as Eqs. (22) and (23). Similar to the above Case B, the variation of the total surface energy $\Delta E_{\text {surface }}$ can be expressed as

$$
\begin{aligned}
\Delta E_{\text {surface }} & =\left(\gamma_{2}+\gamma_{1 L}+\gamma-\gamma_{12}\right) \Delta s+\gamma \varepsilon_{1} \Delta s \\
& =\left(\Delta \gamma+2 \gamma-\Delta \gamma_{1 L}\right) \Delta s+\frac{\gamma(F-\gamma)}{E h} \Delta s .
\end{aligned}
$$

Then energy conservation leads to the governing equation of the peel-off force in this case,

$$
F(1-\cos \alpha)+\frac{(F-\gamma)^{2}}{2 E h}=\gamma_{2}+\gamma_{1 L}+\gamma-\gamma_{12},
$$

which has a very similar form to Eq. (24).

The closed-form solution of the peel-off force in Eq. (30) can be written as

$$
\begin{aligned}
& \frac{F}{E h}=\cos \alpha-1 \\
& \quad+\sqrt{(1-\cos \alpha)^{2}+2 \frac{\Delta \gamma-\Delta \gamma_{1 L}+\gamma(1+\cos \alpha)}{E h}}+\frac{\gamma}{E h} .
\end{aligned}
$$

\section{RESULTS AND DISCUSSION}

It should be noted that, as a special case of the humidity being greater than $90 \%$, i.e., at the vicinity of $\gamma_{1}=\gamma+\gamma_{1 L}$ and $\gamma_{2}=\gamma+\gamma_{2 L}$, if both the limits $\gamma_{1} \rightarrow \gamma+\gamma_{1 L}$ and $\gamma_{2} \rightarrow$ $\gamma+\gamma_{2 L}$ are taken, the peel-off force in the above four different peeling models can be reduced to the same one,

$$
\begin{aligned}
& \frac{F}{E h}=\cos \alpha-1 \\
& \quad+\sqrt{(1-\cos \alpha)^{2}-2 \frac{\gamma(1-\cos \alpha)-\Delta \gamma}{E h}}+\frac{\gamma}{E h},
\end{aligned}
$$

which denotes the continuity among the four peeling models. Furthermore, if the effect of humidity is not considered, the peel-off forces of the four different peeling models can all be well reduced to that of the classical Kendall's model.

The peel-off force as a function of relative humidity is shown in Fig. 4(a) for different peeling angles. Due to some known results of gecko adhesion at different relative humidity, without loss of generality, parameters of the thin film are taken as $E=2 \mathrm{GPa}, h=5 \mathrm{~nm}, A_{H-\text { dry }}=6.5 \times 10^{-20} \mathrm{~J}$, $A_{H-\text { wet }}=3.7 \times 10^{-20} \mathrm{~J}, A_{H-S L}=6.5 \times 10^{-20} \mathrm{~J}$, and $D_{0}=$ $d_{0}=0.2 \mathrm{~nm}, D=d=0.2 \mathrm{~nm}$ according to a single gecko spatula $[19,22,23,37]$. It is shown that, with a determined peeling angle, the peel-off force increases almost linearly
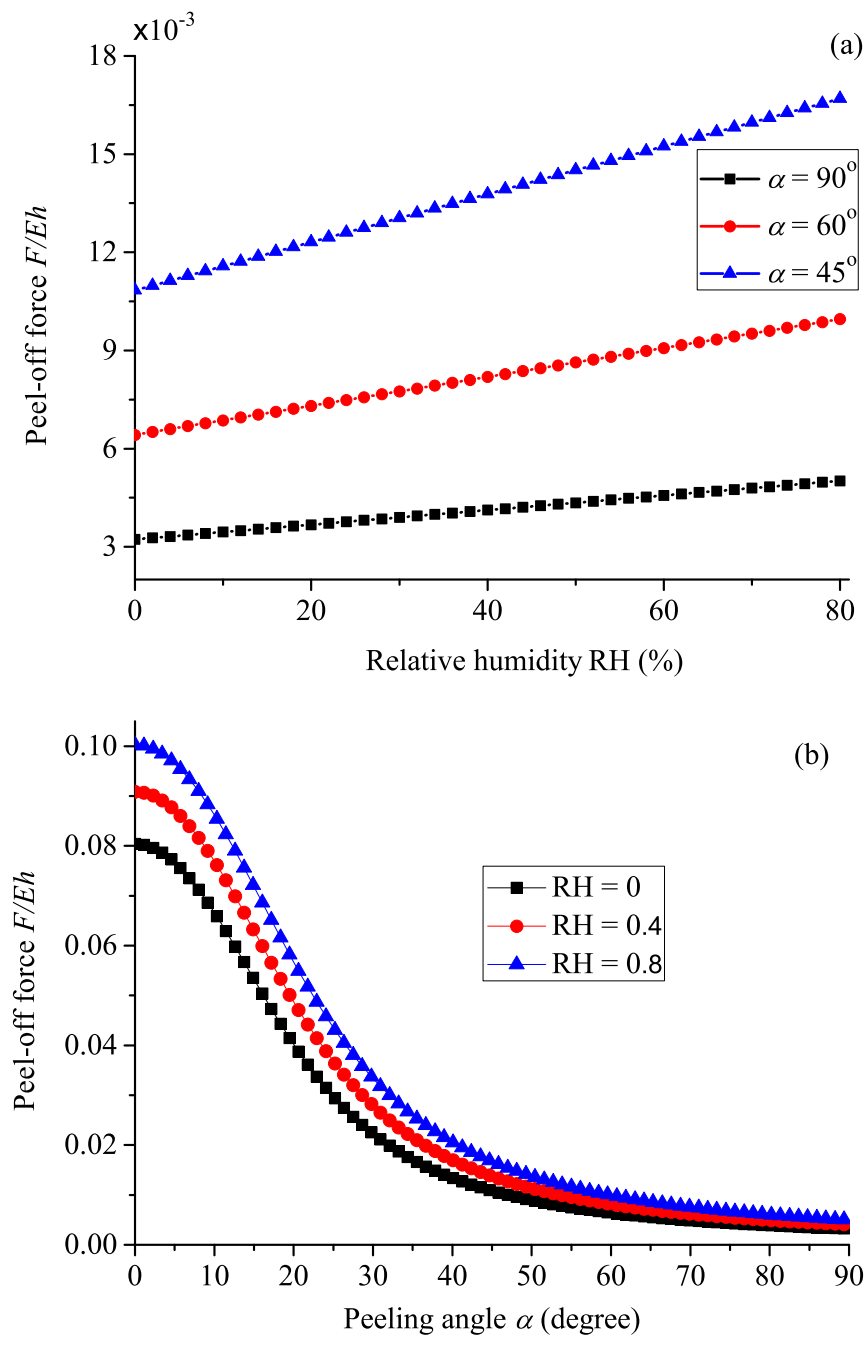

FIG. 4. The peel-off force in the peeling model with an intermediate monolayer water membrane for the relative humidity less than about $90 \%$. (a) The peel-off force as a function of the relative humidity for different peeling angles; (b) the peel-off force varying with the peeling angle for different relative humidities.

with an increasing relative humidity. The relationship between the peel-off force and peeling angle is shown in Fig. 4(b) for different relative humidity, where the result predicted by the Kendall's dry peeling model $(\mathrm{RH}=0)$ is also shown for comparison. With a determined relative humidity, the peel-off force decreases with the increase of peeling angle, which is similar to the result of the Kendall's dry peeling model. Comparing the peel-off force with or without the effect of relative humidity shows that the relative humidity could improve the peel-off force significantly. Although the van der Waals force between the film and substrate is weakened by an intermediate water membrane due to the decreased Hamaker constant, the strong disjoining force between the solid surface and water membrane could compensate and even enhance the total peel-off force.

Although the present model established mainly from the mechanics point of view belongs to a single-level structure and cannot describe the hierarchical adhesion system of gecko strictly, comparison between the theoretical prediction and the 


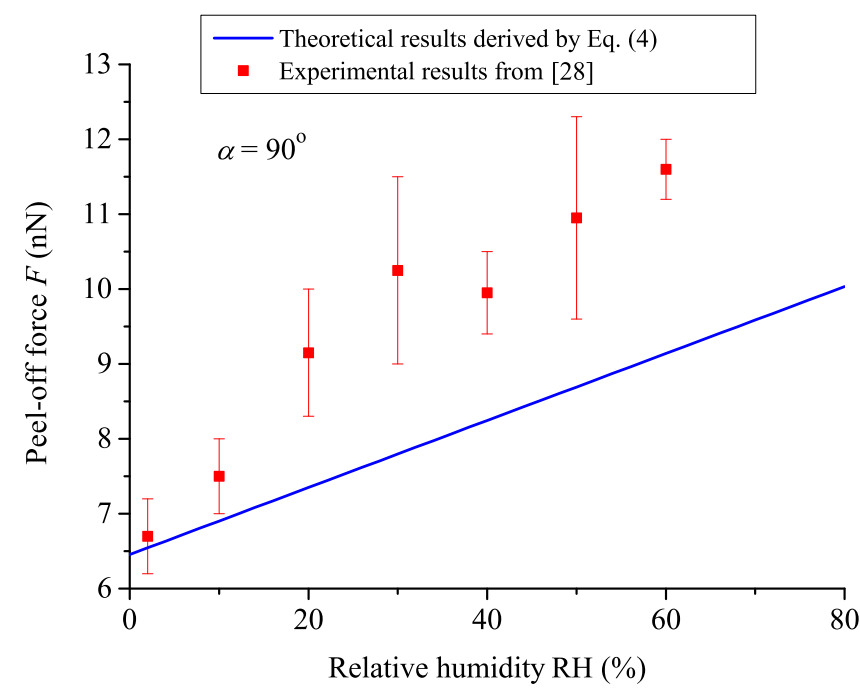

FIG. 5. Comparison of the peel-off force between the experimental measurement done by Huber et al. [28] and the present theoretical prediction, in which the peeling angle is $90^{\circ}$ and the width of the gecko spatula is adopted as $200 \mathrm{~nm}$.

existing experimental results of gecko adhesion at different humidities may give some insights into the mechanical mechanism of gecko adhesion. As for the effect of humidity on gecko adhesion, an alternative theoretical explanation was proposed by Chen and Gao [38], in which they attributed the enhancement of gecko adhesion found in humid environments to the stiffness reduction of seta [38]. Comparison between the experimental measurement for the adhesion force of a single gecko spatula in Huber et al. [28] and the present theoretical prediction is shown in Fig. 5 for the case of a $90^{\circ}$ peeling angle, in which one can see that not only the variation trend but also the order of magnitude of both results agrees with each other. Furthermore, Sun et al. [39] also measured the adhesion force of a single spatula, which was deduced from a hierarchical seta. The adhesion force of a single spatula is from a few nanonewtons to a dozen nanonewtons at different relative humidity [39], which also agrees with the present theoretical prediction. The numerical discrepancy between the theoretical results and the experimental ones may be induced by several factors. The main reason is the measured peel-off force corresponds actually to a two-level hierarchical structure in Huber et al. [28] and Sun et al. [39], which consists of a seta and a spatula. According to Gao et al. [37] and Peng and Chen [24], the peel-off force of a hierarchical structure should be larger than that of a one-level structure. It is the reason that geckos adopt a hierarchical adhesion system to achieve strong adhesion abilities. In addition, the spatula is modeled as a thin film with a uniform thickness in our theoretical work, which is not identical with the shape of a real spatula. The surface roughness of substrates may also be a minor factor. In order to quantitatively explain the experimental results given by Huber et al. [28] and Sun et al. [39], a hierarchical model considering the effect of environmental humidity simultaneously should be developed. Such an innovative work can be carried out in the future.

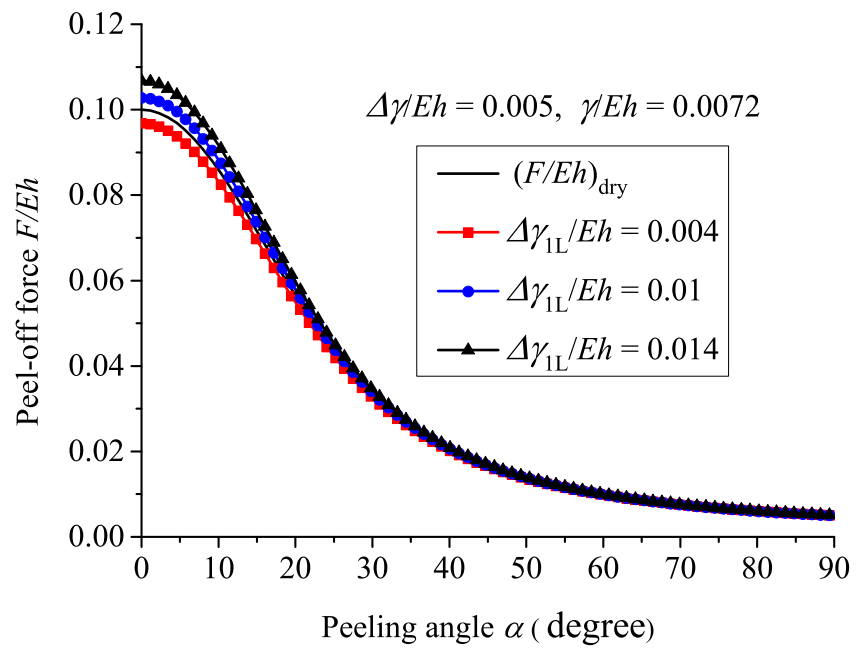

FIG. 6. The peel-off force as a function of the peeling angle with given parameters $\gamma / E h=0.0072, \Delta \gamma / E h=0.005$ and different values of $\Delta \gamma_{1 L} / E h$ in the model of water meniscus condensed near the interface crack tip. The peel-off force derived by the classical Kendall's model is also shown for comparison.

In the case of a water meniscus condensed near the interface crack tip as shown in Fig. 2(a), Eq. (20) demonstrates that the peel-off force is influenced by the peeling angle $\alpha$, the interface adhesion energy between the film and substrate $\Delta \gamma$, the interface energy between water meniscus and film $\Delta \gamma_{1 L}$, and the surface tension of water $\gamma$. Figure 6 gives a typical result predicted by Eq. (20), where the peel-off force varies with the peeling angle for a determined water surface tension $\gamma / E h=0.0072$ and interface energy between the film and substrate $\Delta \gamma / E h=0.005$. The result obtained by the classical Kendall's model [5] is also plotted for comparison. From Fig. 6, one can see that the water meniscus condensed at the interface crack tip has a significant and complicated effect on the peel-off force. In general, the peel-off force for both the dry and wet peeling models decreases with an increasing peeling angle. At a given peeling angle, the peel-off force increases with an increasing interface energy $\Delta \gamma_{1 L} / E h$. Comparing with the dry peel-off force predicted by the classical Kendall's model, the wet peel-off force is either larger or smaller than the dry one depending on the value of $\Delta \gamma_{1 L} / E h$.

When the water droplet spreads on the film and substrate surfaces as shown in Fig. 2(b), the peel-off force depends on several physical parameters, such as the interface energy of the water membrane and film, the interface energy of the water membrane and substrate, the surface tension of water, the peeling angle, and the interface adhesion energy of the film and substrate. Figure 7(a) gives the peel-off force as a function of the peeling angle for a given water surface tension $\gamma / E h=0.0072$, a determined interface adhesion energy of the film and substrate $\Delta \gamma / E h=0.005$, and a fixed interface energy of the water membrane and film $\Delta \gamma_{1 L} / E h=0.0145$, but for different adhesion energies of the water membrane and substrate $\Delta \gamma_{2 L} / E h$. Equation (25) demonstrates that the effect of $\Delta \gamma_{1 L} / E h$ on the peel-off force is similar to that of $\Delta \gamma_{2 L} / E h$. Only the effect of $\Delta \gamma_{2 L} / E h$ on the peel-off force is analyzed in this model. The peel-off force predicted by 

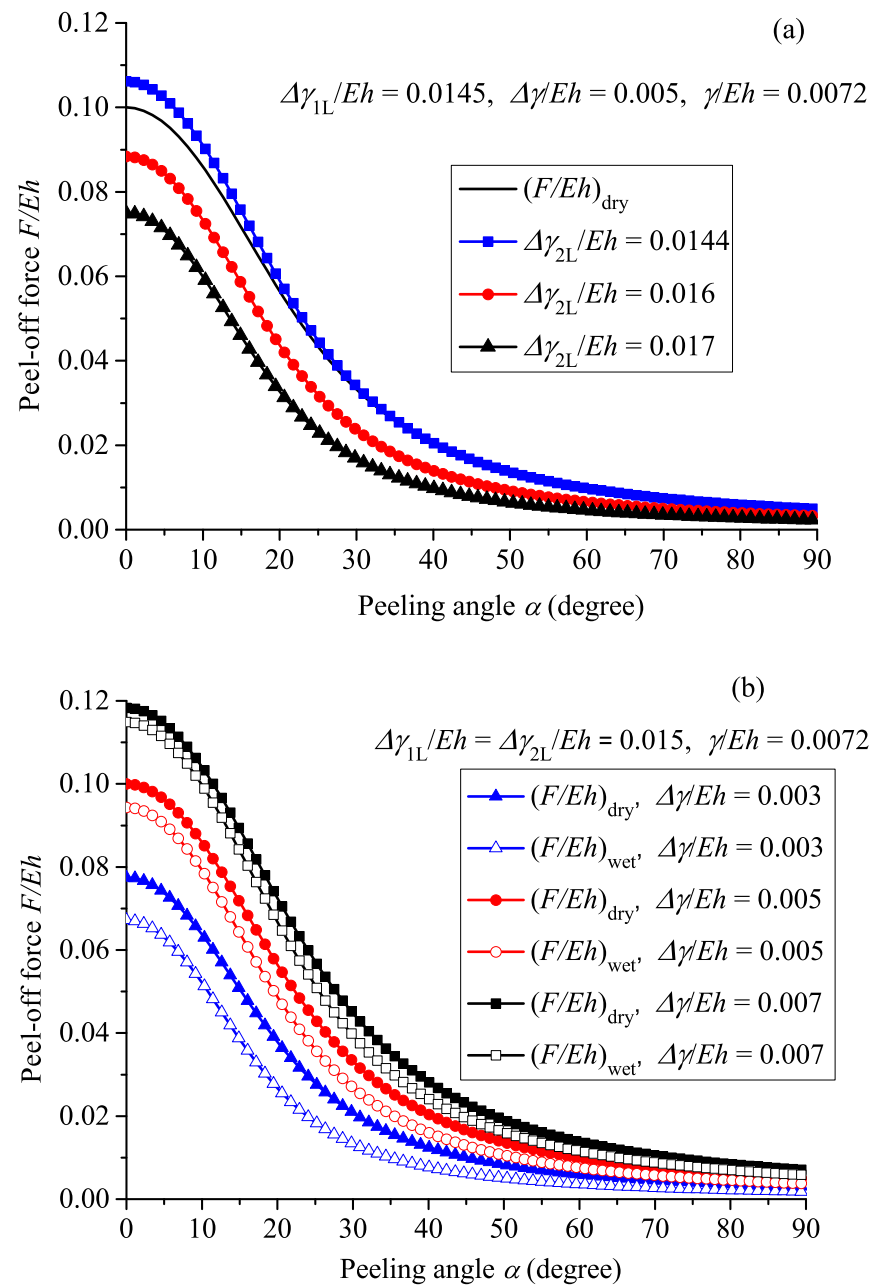

FIG. 7. The peel-off force in the peeling model with water droplets spreading on solid surfaces. (a) The peel-off force varying with the peeling angle for determined parameters $\gamma / E h=0.0072$, $\Delta \gamma / E h=0.005$, and $\Delta \gamma_{1 L} / E h=0.0145$, but for different values of $\Delta \gamma_{2 L} / E h$; (b) the peel-off force varying with the peeling angle for determined parameters $\Delta \gamma_{1 L} / E h=\Delta \gamma_{2 L} / E h=0.015$, $\gamma / E h=0.0072$, but for different interface adhesion energies of the film and substrate $\Delta \gamma / E h$, where the result predicted by the classical Kendall's dry peeling model is shown for comparison.

the classical Kendall's model is also shown in Fig. 7(a) for comparison, which only depends on the peeling angle and the interface adhesion energy between the film and substrate. Figure 7(a) shows that the spreading water membrane also has a significant effect on the peel-off force. The peel-off force decreases with the increase of the peeling angle. Different from the case A, the peel-off force decreases with an increasing interface energy $\Delta \gamma_{2 L} / E h$ at a given peeling angle. Comparing to the dry peel-off force predicted by the classical Kendall's model, the water capillarity can also either enhance or reduce the peel-off force. At the critical condition of $\Delta \gamma_{2 L}=2 \gamma$, the wet peel-off force is larger than the dry one due to the effect of water surface tension as shown in Eq. (25).

Figure 7(b) gives the effect of interface adhesion energy of the film and substrate on the wet peel-off force with determined wet-surface parameters $\gamma / E h=0.0072$ and
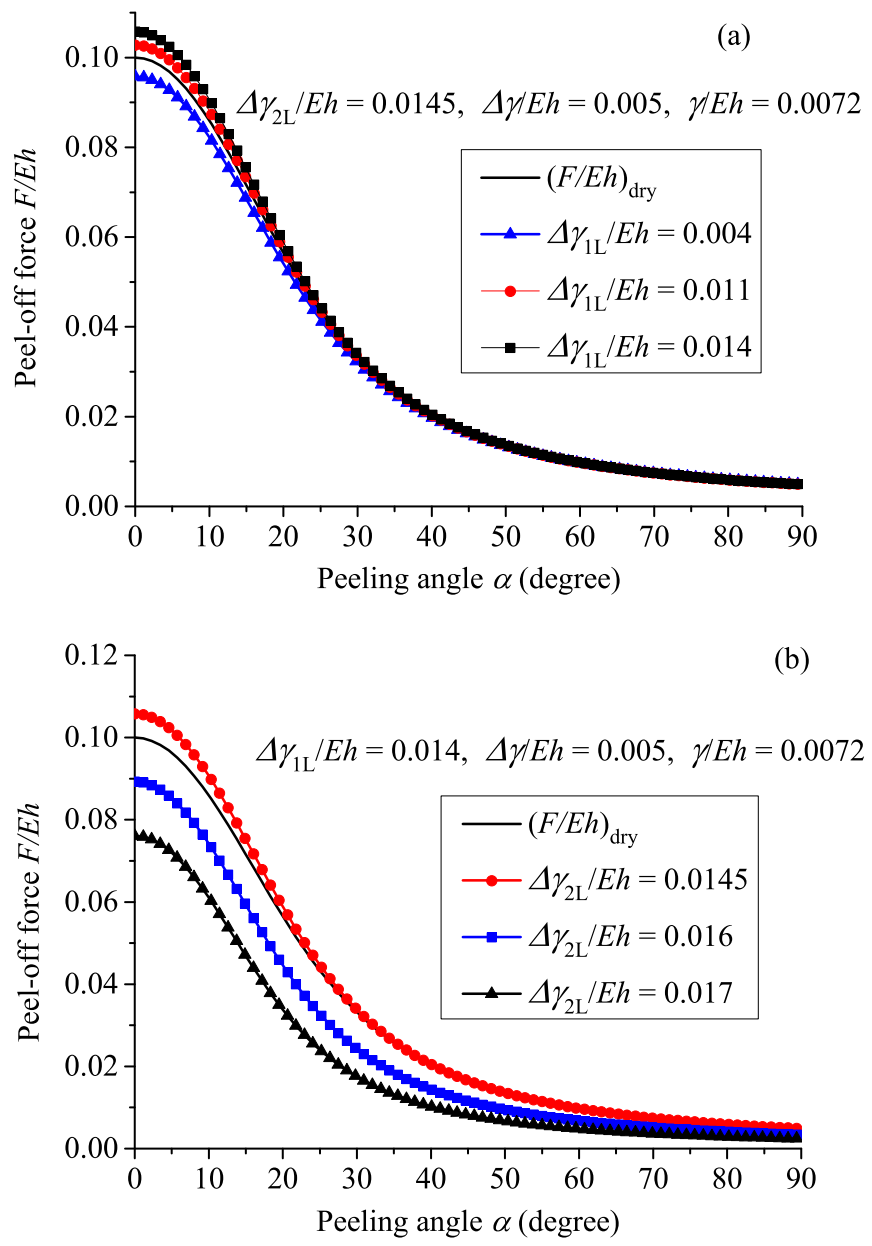

FIG. 8. The peel-off force in the model of the water droplet being pinned on the film surface but completely spreading on the substrate surface. (a) The peel-off force varying with the peeling angle for determined parameters $\gamma / E h=0.0072, \Delta \gamma / E h=0.005$, and $\Delta \gamma_{2 L} / E h=0.0145$, but for different values of $\Delta \gamma_{1 L} / E h$; (b) the peeloff force varying with the peeling angle for determined parameters $\gamma / E h=0.0072, \Delta \gamma / E h=0.005$, and $\Delta \gamma_{1 L} / E h=0.014$, but for different values of $\Delta \gamma_{2 L} / E h$.

$\Delta \gamma_{1 L} / E h=\Delta \gamma_{2 L} / E h=0.015$, where the dry peel-off force is also shown for comparison. Figure 7(b) exhibits that the wet peel-off force increases with an increasing interface adhesion energy of the film and substrate $\Delta \gamma / E h$ for a determined peeling angle, which is very similar to the result predicted by the Kendall's dry peeling model.

When the water droplet partially wets the film surface and completely spreads on the surface of the substrate as shown in Fig. 2(c), the effect of the water meniscus on the peel-off force is shown in Fig. 8, where the peel-off force achieved by the classical Kendall's model, i.e., a dry peeling model, is also given for comparison. It is found that the wet peel-off force has a similar varying trend to that of the dry one. The peel-off force decreases with an increasing peeling angle. One should note that, for the fixed values of parameters $\gamma / E h=0.0072, \Delta \gamma / E h=0.005$, the interface energies $\Delta \gamma_{1 L} / E h$ and $\Delta \gamma_{2 L} / E h$ have a different influence on the peel-off force due the different wetting configurations of water on the thin film and substrate. The peel-off force 


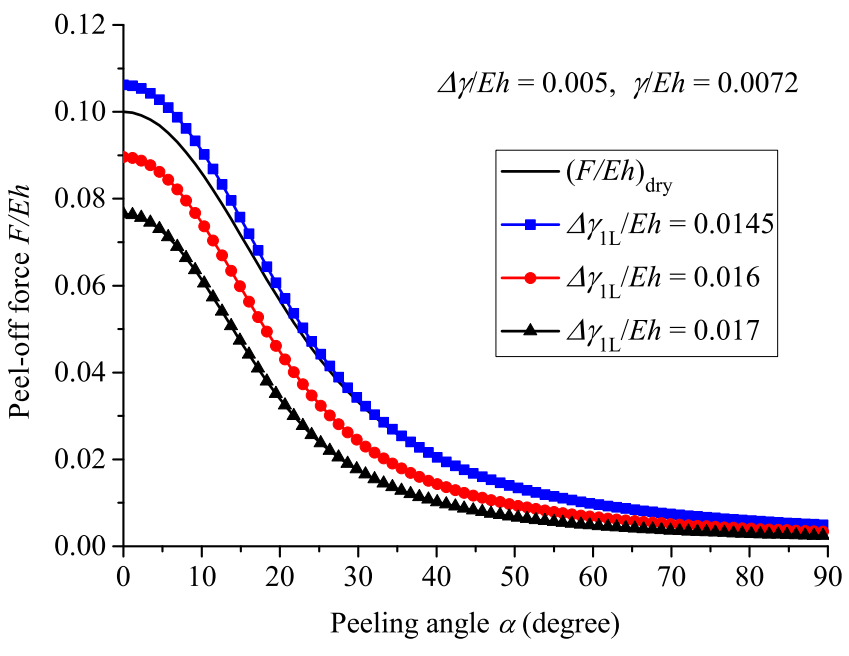

FIG. 9. The peel-off force varying with the peeling angle with $\Delta \gamma / E h=0.005, \gamma / E h=0.0072$, but different values of $\Delta \gamma_{1 L} / E h$ in the model of water droplet pinned on the substrate surface but completely spreading on the surface of the film.

increases with the interface energy $\Delta \gamma_{1 L} / E h$ increasing, but decreases with the increasing interface energy $\Delta \gamma_{2 L} / E h$ at a given peeling angle as shown in Figs. 8(a) and 8(b). Comparing with the dry peeling model, the wetting peel-off force can be either enhanced or reduced by the water meniscus depending on the interface energy between the water and solid surface.

When the water droplet partially wets the substrate surface and completely spreads on the surface of the film as shown in Fig. 2(d), the peel-off force as a function of the peeling angle is shown in Fig. 9 with a given water surface tension $\gamma / E h=0.0072$ and a fixed adhesion energy between the film and substrate $\Delta \gamma / E h=0.005$. One can see that the peel-off force decreases with the peeling angle increasing. But at a given peeling angle, the wet peel-off force decreases with the interface energy $\Delta \gamma_{1 L} / E h$ increasing, which is similar to that of case B.

\section{CONCLUSION}

Inspired by gecko adhesion in humid environments, we extended the classical Kendall's peeling model to a wet one by introducing the effect of relative humidity. According to the natural phenomenon that a monolayer of water molecules may be adsorbed on surfaces for the relative humidity less than about $90 \%$, and otherwise water droplets may be condensed, we established two kinds of wet peeling models. One corresponds to a monolayer of water membrane existing between the film and the substrate as an intermediate layer and the other relates to a water droplet near the interfacial crack tip. In the former, the peel-off force is significantly improved by the monolayer of water membrane due to the strong disjoining pressure. In the latter, water droplets may form a water meniscus near the interface crack tip with four wetting cases as shown in Fig. 2, depending on the competition of surface tension of water, the interface energy of the film and water, and the interface energy of the substrate and water. In this case, the peel-off force is seriously affected by the capillary force, which is either larger or smaller than the dry one predicted by the classical Kendall's model, depending on the adhesion energy of the solid surface and water. The limitation condition among the four different peeling models shown in Fig. 2 indicates that all four models are continuous at the vicinity of the wetting transition. All the results should be useful not only for understanding the bioadhesion mechanism in humid environments, but also for assessing the interface behavior of film-substrate systems in practical applications.

\section{ACKNOWLEDGMENTS}

The work reported here is supported by NSFC through Grants No. 11302228, No. 11372317, and No. 11532013, and the 973 Nano-project (2012CB937500). We appreciate the anonymous reviewers for their valuable suggestions.
[1] G. J. Spies, Aircr. Eng. Aerosp. Technol. 25, 64 (1953).

[2] J. Kim, K. S. Kim, and Y. H. Kim, J. Adhes. Sci. Technol. 3, 175 (1989).

[3] R. A. Sauer, J. Adhes. 87, 624 (2011).

[4] A. J. Kinloch, C. C. Lau, and J. G. Williams, Int. J. Fract. 66, 45 (1994).

[5] K. Kendall, J. Phys. D: Appl. Phys. 8, 1449 (1975).

[6] K. S. Kim and N. Aravas, Int. J. Solids Struct. 24, 417 (1988).

[7] Y. Wei and J. W. Hutchinson, Int. J. Fract. 93, 315 (1998).

[8] M. D. Thouless and H. M. Jensen, J. Adhes. 38, 185 (1992).

[9] M. J. Loukis and N. Aravas, J. Adhes. 35, 7 (1991).

[10] H. Chen, X. Feng, Y. Huang, Y. G. Huang, and J. A. Rogers, J. Mech. Phys. Solids 61, 1737 (2013).

[11] Z. L. Peng, C. Wang, L. Chen, and S. H. Chen, Int. J. Solids Struct. 51, 4596 (2014).

[12] S. Xia, L. Ponson, G. Ravichandran, and K. Bhattacharya, Phys. Rev. Lett. 108, 196101 (2012).
[13] S. M. Xia, L. Ponson, G. Ravichandran, and K. Bhattacharya, J. Mech. Phys. Solids 61, 838 (2013).

[14] Z. L. Peng and S. H. Chen, Phys. Rev. E 91, 042401 (2015).

[15] Z. L. Peng and S. H. Chen, Int. J. Solids Struct. 60-61, 60 (2015).

[16] X. Oyharcabal and T. Frisch, Phys. Rev. E 71, 036611 (2005).

[17] Z. X. Lu, S. W. Yu, X. Y. Wang, and X. Q. Feng, Eur. Phys. J. E 23, 67 (2007).

[18] M. D. Thouless and Q. D. Yang, Int. J. Adhes. Adhes. 28, 176 (2008).

[19] Y. Tian, N. Pesika, H. B. Zeng, K. Rosenberg, B. X. Zhao, P. McGuiggan, K. Autumn, and J. Israelachvili, Proc. Natl. Acad. Sci. U. S. A. 103, 19320 (2006).

[20] N. S. Pesika, Y. Tian, B. X. Zhao, K. Rosenberg, H. B. Zeng, P. McGuiggan, K. Autumn, and J. N. Israelachvili, J. Adhes. 83, 383 (2007).

[21] Z. L. Peng, S. H. Chen, and A. K. Soh, Int. J. Solids Struct. 47, 1952 (2010). 
[22] Z. L. Peng and S. H. Chen, Colloids Surf., B 88, 717 (2011).

[23] Z. L. Peng and S. H. Chen, Phys. Rev. E 83, 051915 (2011).

[24] Z. L. Peng and S. H. Chen, Appl. Phys. Lett. 101, 163702 (2012).

[25] K. Autumn, Y. A. Liang, S. T. Hsieh, W. Zesch, W. P. Chan, T. W. Kenny, R. Fearing, and R. J. Full, Nature 405, 681 (2000).

[26] K. Autumn, M. Sitti, Y. A. Liang, A. M. Peattie, W. R. Hansen, S. Sponberg, T. W. Kenny, R. Fearing, J. N. Israelachvili, and R. J. Full, Proc. Natl. Acad. Sci. U. S. A. 99, 12252 (2002).

[27] B. Chen, P. D. Wu, and H. J. Gao, J. R. Soc., Interface 6, 529 (2009).

[28] G. Huber, H. Mantz, R. Spolenak, K. Mecke, K. Jacobs, S. N. Gorb, and E. Arzt, Proc. Natl. Acad. Sci. U. S. A. 102, 16293 (2005).

[29] F. W. DelRio, M. L. Dunn, and M. P. de Boer, Scr. Mater. 59, 916 (2008).

[30] C. M. Mate and V. J. Novotny, J. Chem. Phys. 94, 8420 (1991).
[31] C. M. Mate, J. Appl. Phys. 72, 3084 (1992).

[32] N. Tas, T. Sonnenberg, H. Jansen, R. Legtenberg, and M. Elwenspoek, J. Micromech. Microeng. 6, 385 (1996).

[33] C. H. Mastrangelo and C. H. Hsu, J. Microelectromech. Syst. 2, 33 (1993).

[34] J. N. Israelachvili, Intermolecular and Surface Forces (Academic Press, London, 1992).

[35] A. G. Evans, M. Ruhle, B. J. Dalgleish, and P. G. Charalambides, Mater. Sci. Eng., A 126, 53 (1990).

[36] J. W. Hutchinson and Z. Suo, Adv. Appl. Mech. 29, 63 (1992).

[37] H. J. Gao, X. Wang, H. M. Yao, S. Gorb, and E. Arzt, Mech. Mater. 37, 275 (2005).

[38] M. Prowse, M. Wilkinson, J. Puthoff, G. Mayer, and K. Autumn, Acta Biomater. 7, 733 (2011).

[39] W. X. Sun, P. Neuzil, T. S. Kustandi, S. Oh, and V. D. Samper, Biophys. J. 89, L14 (2005). 